\title{
Gangliocytic Paraganglioma with Carcinoma of the Ampulla of Vater
}

\author{
Masanari Sekine, Hiroyuki Miyatani, Keita Matsumoto, Hitomi Kashima, Yudai Koito, \\ Takaya Miura, Yuko Takahashi, Rumiko Tsuboi, Takehiro Ishii, Junichi Fujiwara, \\ Takeshi Uehara, Shunsuke Urayoshi, Kazuhito Yuhashi, Takeharu Asano, Noriyoshi Sagihara, \\ Satohiro Matsumoto and Hirosato Mashima
}

\begin{abstract}
:
The patient was a "73"-year-old woman who visited our hospital with the chief complaint of weight loss. Upper gastrointestinal endoscopy revealed an enlarged ampulla of Vater, and a biopsy led to a diagnosis of Group "4" gastric carcinoma; suspicious of adenocarcinoma. There were no findings suggesting invasion into the muscle layer of duodenum, despite tumor mass formation being observed in the sphincter of Oddi. We performed endoscopic papillectomy for both diagnostic and therapeutic purposes. Pathologically, a welldifferentiated adenocarcinoma existed in the superficial layer of the mucous membrane of the papilla of Vater, and gangliocytic paraganglioma was present in the deep portion. The resected margins of both lesions were negative.
\end{abstract}

Key words: gangliocytic paraganglioma, ampulla of Vater, carcinoma, endoscopic papillectomy

(Intern Med 57: 2663-2668, 2018)

(DOI: 10.2169/internalmedicine.0464-17)

\section{Introduction}

Gangliocytic paraganglioma (GP) is a rare tumor that occurs mainly in the descending part of the duodenum (1). GP is often recognized as a submucosal tumor by upper gastrointestinal endoscopy, but making a definitive diagnosis is considered to be difficult. Surgery has been performed to treat ampulla of Vater tumors, and in particular, carcinoma of the ampulla of Vater. Surgery requires pancreaticoduodenectomy, but a less invasive transduodenal papillectomy is selected in some cases. A more limited surgery is endoscopic papillectomy. This endoscopic technique is currently widely used, but it is only indicated for adenoma. The indications have recently been expanded to superficial cancer, but diagnosis of the stage of cancer progression is difficult, and risks such as residual carcinoma and recurrence should be taken into consideration when planning treatment approaches. GP of the papilla of Vater has been reported, but there are no reports describing it to be complicated with am- pullary carcinoma. We herein report a case with carcinoma of the ampulla of Vater coexisting with GP, which was diagnosed after papillectomy.

\section{Case Report}

The patient was a "73"-year-old woman who visited another hospital before coming to us with a chief complaint of "5" kg weight loss. A tumor of the ampulla of Vater was suspected by computerized tomography (CT) and gastrointestinal endoscopy was performed, revealing an enlargement of the papilla of Vater (Fig. 1). The patient was referred to our hospital for further examination. Regarding the patient's family history, her father had lung cancer and her sister had multiple myeloma. Her past medical history was unremarkable. Blood tests revealed no abnormalities in blood count or biochemical examinations, with both carcinoembryonic antigen (CEA) and CA"19"-"9" being in the normal range (Table). Upper gastrointestinal endoscopy and a biopsy were also performed at our hospital, but there were no findings 

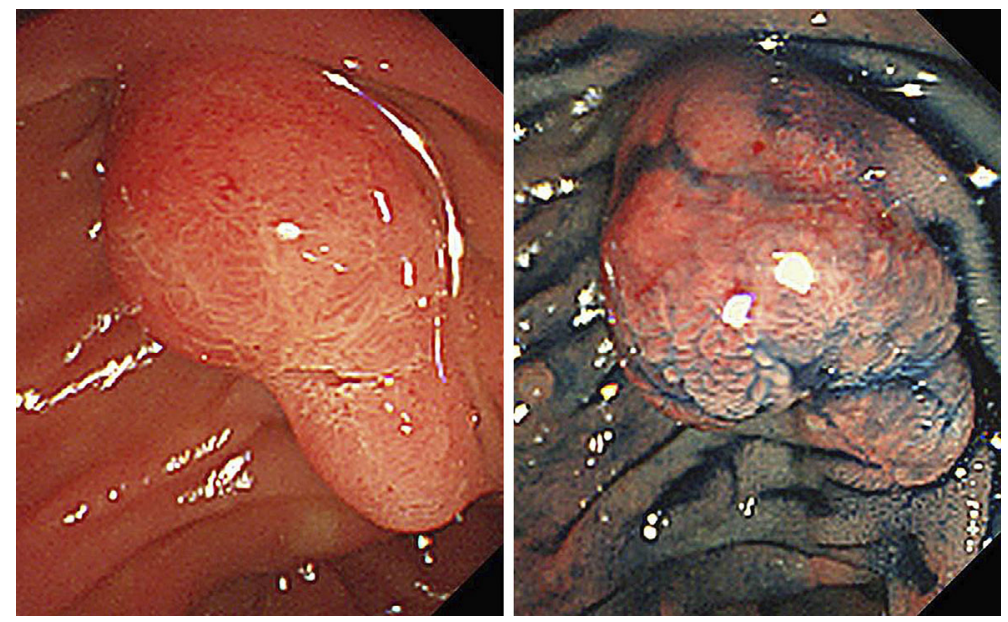

Figure 1. Endoscopic image of the papilla of Vater. The papilla of Vater was slightly tense and swollen, and the oral side was slightly reddish. After applying indigo carmine, erythema was seen on the oral side of the papilla of Vater.

Table. Laboratory Data.

\begin{tabular}{lclclr}
\hline \multicolumn{1}{l}{ Blood count } & & \multicolumn{4}{l}{ Biochemical examination } \\
WBC & $5,490 / \mathrm{mL}$ & Alb & $4.3 \mathrm{~g} / \mathrm{dL}$ & BUN & $21 \mathrm{mg} / \mathrm{dL}$ \\
$\mathrm{Hb}$ & $14.7 / \mathrm{dL}$ & T.bil & $0.9 \mathrm{mg} / \mathrm{dL}$ & $\mathrm{Cre}$ & $0.65 \mathrm{mg} / \mathrm{dL}$ \\
$\mathrm{Plt}$ & $194,000 / \mathrm{mL}$ & AST & $22 \mathrm{IU} / \mathrm{L}$ & $\mathrm{Na}$ & $139 \mathrm{mEq} / \mathrm{L}$ \\
& & ALT & $18 \mathrm{IU} / \mathrm{L}$ & $\mathrm{K}$ & $3.8 \mathrm{mEq} / \mathrm{L}$ \\
\multicolumn{1}{l}{ Tumor marker } & & ALP & $266 \mathrm{IU} / \mathrm{L}$ & $\mathrm{Cl}$ & $103 \mathrm{mEq} / \mathrm{L}$ \\
CEA & $1.7 \mathrm{ng} / \mathrm{mL}$ & $\gamma-\mathrm{GTP}$ & $16 \mathrm{IU} / \mathrm{L}$ & $\mathrm{CRP}$ & $0.02 \mathrm{mg} / \mathrm{dL}$ \\
CA19-9 & $11.5 \mathrm{U} / \mathrm{mL}$ & AMY & $84 \mathrm{IU} / \mathrm{L}$ & & \\
& & P-AMY & $30 \mathrm{IU} / \mathrm{L}$ & & \\
\hline
\end{tabular}

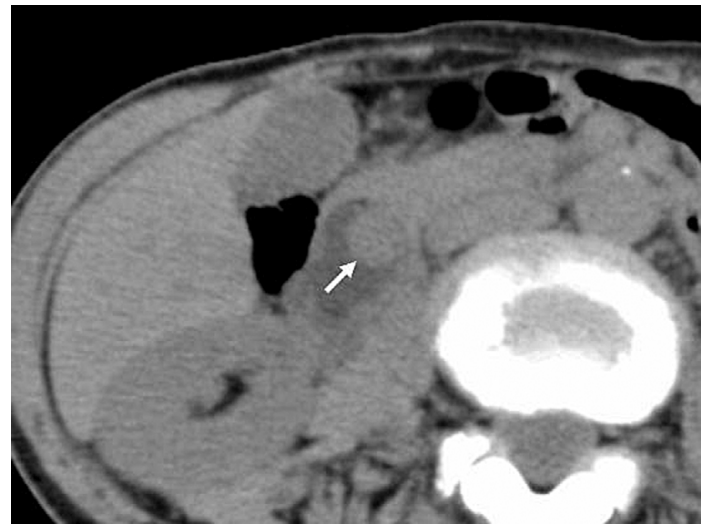

Figure 2. CT image (non-contrast-enhanced CT) of the papilla of Vater. An enlarged overhanging papilla of Vater was observed in the duodenum (arrow).

indicative of malignancy. Reexamination was performed " 3 " months later. The biopsy at that time yielded a diagnosis of "Group "4" gastric carcinoma, well-differentiated tubular adenocarcinoma suspected" (Japanese Classification of Gastric Carcinoma) (2). An enlargement of the papilla of Vater was suspected, but no obvious lymph node metastasis was noted in the CT study (Fig. 2). Endoscopic ultrasonography (EUS) confirmed an enlarged papilla of Vater, which was an isoechoic and hypoechoic tumor mass, measuring " 12 "-mm in maximum diameter, but the muscle layer was well maintained. No enlargement was observed in either the bile duct or the pancreatic duct, with no findings suggestive of obvious tumor progression were observed (Fig. 3). Endoscopic retrograde cholangiopancreatography (ERCP) showed neither any irregularity nor enlargement of either the bile duct or the pancreatic duct (Fig. 4). Intraductal ultrasonography (IDUS) also showed the bile duct and the pancreatic duct to be free of tumor invasion. There were no cytological findings indicative of malignancy in either the patient's bile or pancreatic juice. Although exposed carcinoma of the ampulla of Vater, stage T"1", was suspected and thus pancreatoduodenectomy as a standard treatment was considered, endoscopic papillectomy was instead selected for diagnostic purposes as the patient declined more invasive surgery. A “20”-mm snare and VIO “300”D endoscope (ERBE, Tübingen, Germany) were used. After resection with End Cut mode ("80"W, Effect"3" Endo cut mode Q) was performed, a clip was placed at the frenulum, and a "7"Fr plastic stent ("5" $\mathrm{cm}$ ) and a "5"Fr plastic stent ("4" $\mathrm{cm}$ ) were placed in the bile duct and the pancreatic duct, respectively (Fig. 5). Her subsequent clinical course was good. A histopathological examination showed growth of the mucosal epithelium with obvious stacking of nuclei on the superficial layer. 

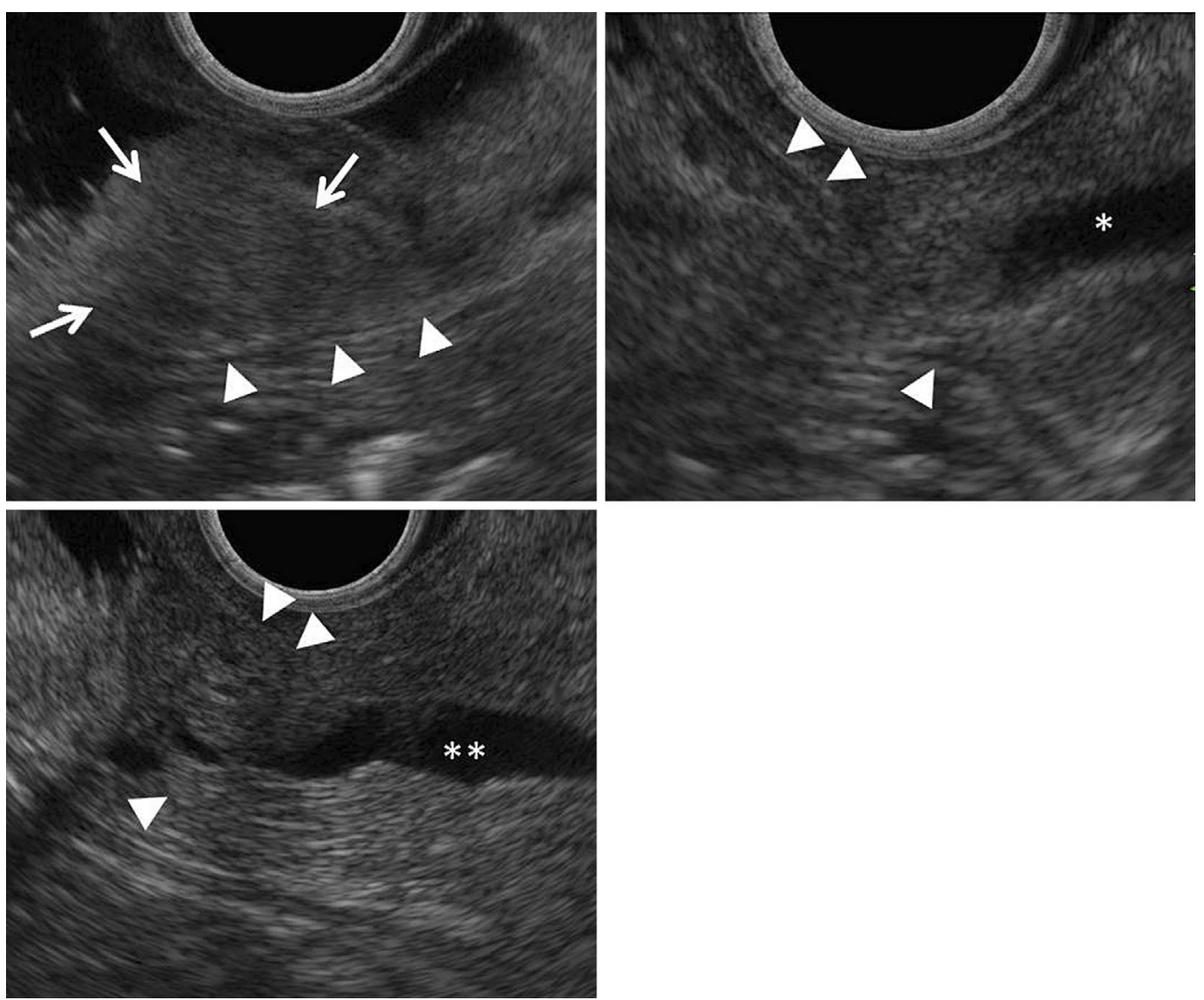

Figure 3. EUS images of the papilla of Vater. A homogeneous and hypoechoic tumor mass, measuring 12-mm in maximum diameter, was observed (arrow) and the muscle layer of the duodenum was maintained (arrowhead). The muscle of the papilla of Vater was preserved and no tumor progression was evident in either the bile duct $(*)$ or the pancreatic duct $(* *)$.
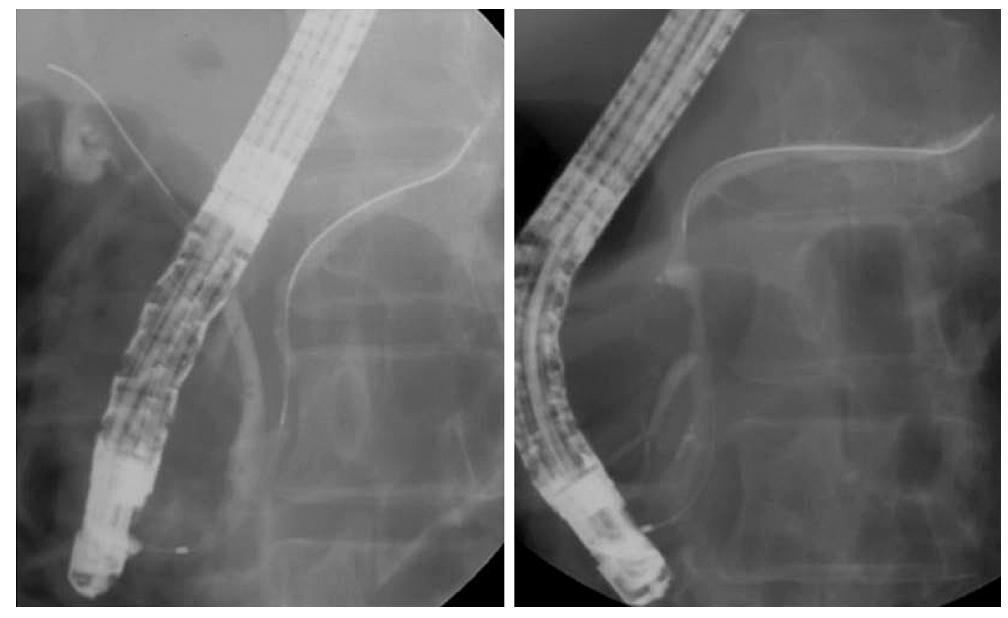

Figure 4. ERCP images. No dilation was observed in either the bile duct or the pancreatic duct. IDUS, similarly, revealed no evidence of tumor progression in either the bile duct or the pancreatic duct.

Most of the specimen showed cytological atypia corresponding to adenoma (Fig. 6a and b), but there were some strongly atypical glandular ducts, demonstrating findings suggestive of well-differentiated tubular adenocarcinoma (Fig. 6a and c). The resected margin was negative for malig- nant cells. In the deep portion, there were proliferative cell clumps forming folliculi (Fig. 6d-f). Immunostaining of the cell clumps were CD"56"-positive, synaptophysin-positive, neuron-specific enolase (NSE)-negative, cytokeratin (AE"1"/ AE"3")-positive, S"100"-positive, and chromogranin- 

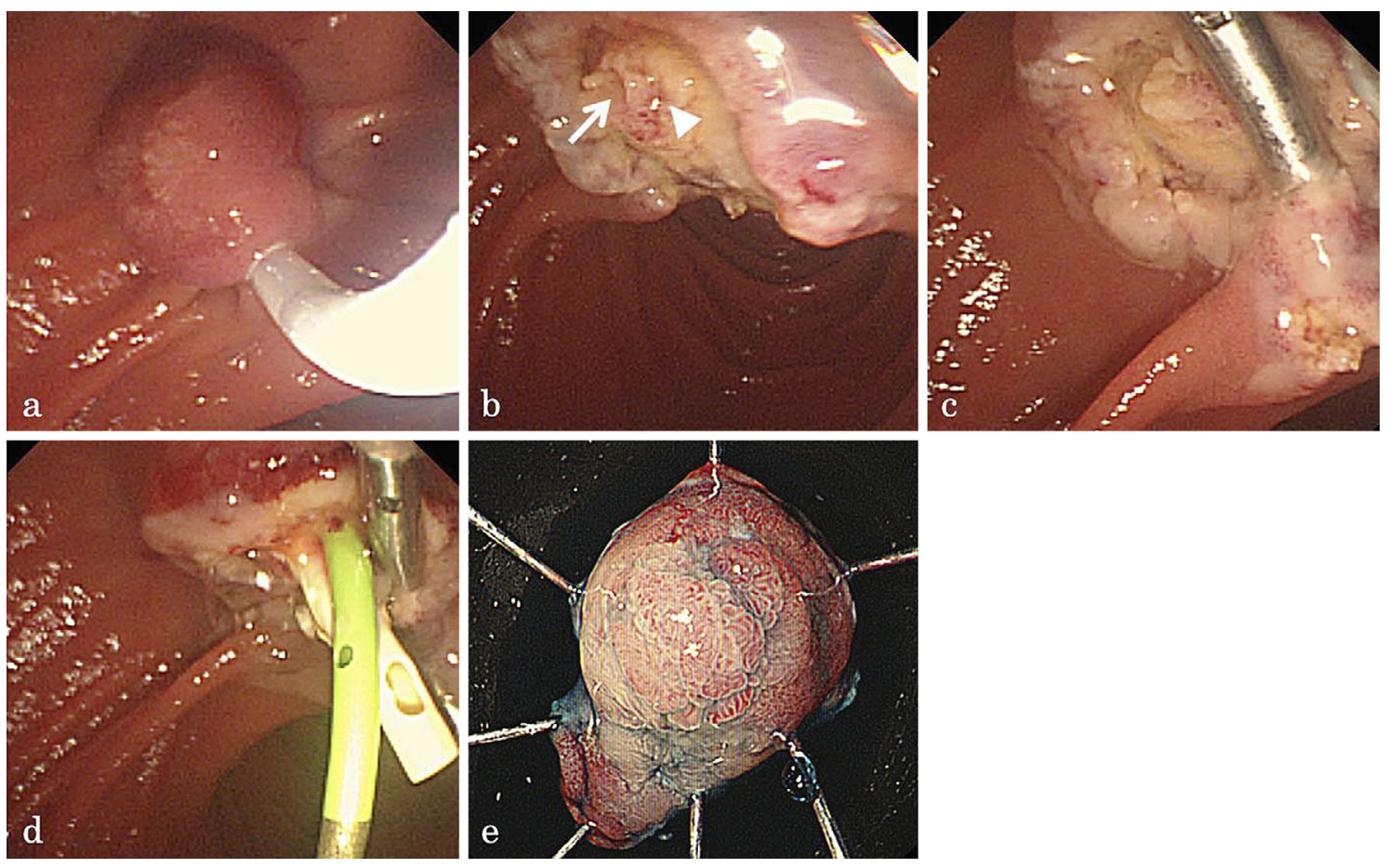

Figure 5. Procedure of endoscopic papillectomy. a: The snare was squeezed around the target in the direction from the oral side to the anal side. b: After resection, the openings of the bile duct (arrow) and the pancreatic duct (arrowhead) could be confirmed. c: The frenulum was clipped for closure. d: A stent was placed in the bile duct and the pancreatic duct. e: The resected specimen: Macroscopically, an en bloc resection was achieved.
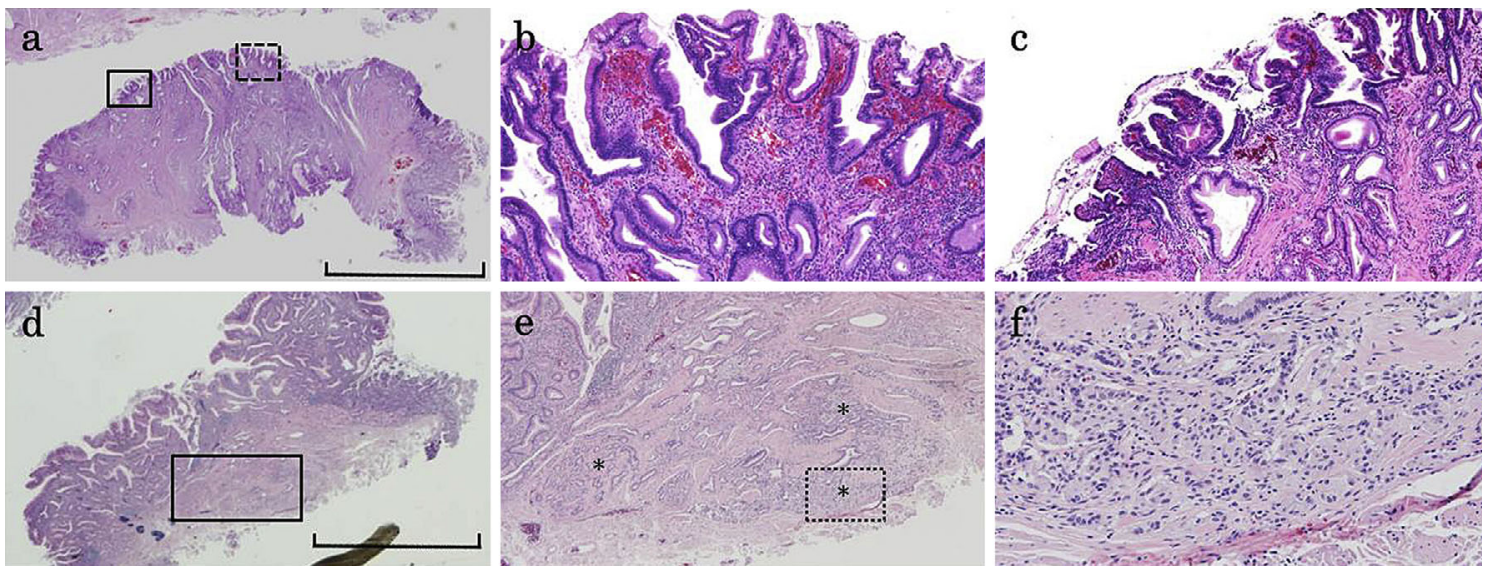

Figure 6. Pathological images of the resected specimen in Hematoxylin and Eosin staining. a: A loupe image of the cancer site, b: Magnification of the outlined square showed the dotted line in a. There was growth of the mucosal epithelium with an obvious stacking of nuclei. Most of the specimen showed cytological atypia corresponding to adenoma, $c$ : Magnification of the outlined square showed the solid line in a. There were some strongly atypical gland ducts, which were consistent with adenocarcinoma. d: A loupe image of gangliocytic paraganglioma, e: Magnification of the outlined square showed the solid line in d: Cell clumps proliferating to form folliculi $(*)$ were seen. $f$ : Magnification of the outlined square dotted line in e. Bar=3 mm.

negative, leading to the diagnosis of GP. The margin of the deeply resected tissue was negative (Fig. 7). Follow-up observation with upper gastrointestinal endoscopy, EUS and $\mathrm{CT}$ is planned for this patient.

\section{Discussion}

GP is a rare tumor usually developing in the ampulla of Vater and adjacent area, as well as the descending part of 


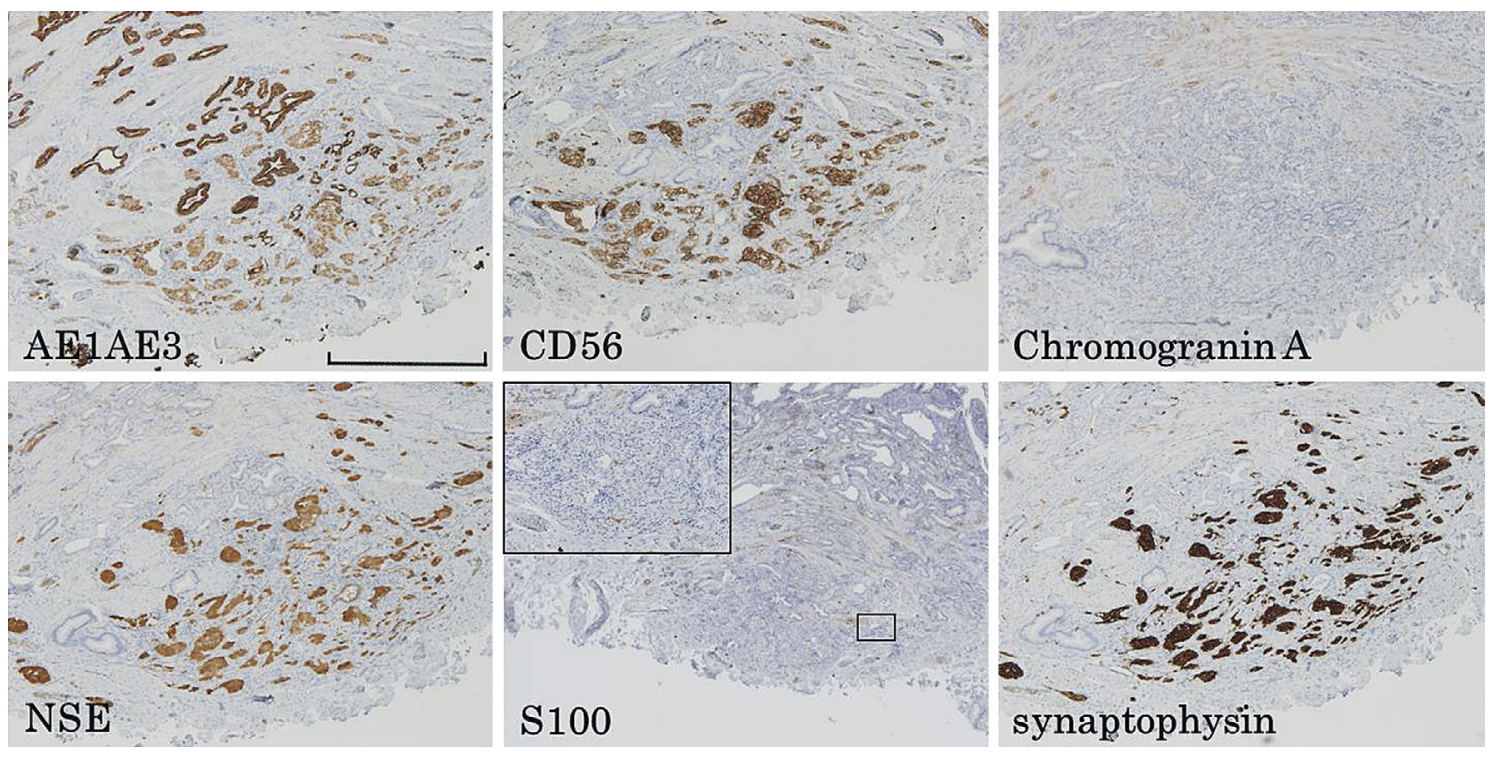

Figure 7. Immunostaining of gangliocytic paraganglioma. Immunostaining results were AE1AE3positive, CD56-positive, Chromogranin A-negative, NSE-positive, S100-positive (Magnification of the outlined square), and Synaptophysin-positive, leading to the diagnosis of GP. Bar=150 $\mu \mathrm{m}$.

the duodenum. This tumor was reported as ganglioneuroma of the digestive tract for the first time in "1957" by Dahl et al., and it had three distinct cellular components, i.e., epithelioid, spindle, and ganglion-like cells $(1,3,4)$. Terms such as non-chromaffin, paraganglioma, paraganglioneuroma, and gangliocytoma have been used based on the main component, but currently, GP, as proposed by Kepes et al., is generally used. This tumor reportedly occurs in men slightly more frequently than in women (5). The symptoms include gastrointestinal bleeding and abdominal pain, but many cases are considered to be asymptomatic. It has a form similar to a submucosal tumor and on occasion shows erosion and ulceration on the surface (6). A definitive diagnosis is often not made because confirming the three aforementioned pathological cell types is difficult. Although GP is generally considered to be benign, there are reports describing cases with lymph node metastasis and distant metastasis, and therefore the resection method and follow-up period should be carefully determined (7-9). When we searched PubMed with the key words "gangliocytic paraganglioma," "ampulla of Vater," "carcinoma," and "cancer," there were no reports describing any cases with this complication.

GP presents a variety of imaging findings, making a definitive preoperative diagnosis difficult. GP has the form of a submucosal tumor, and EUS is useful for distinguishing it from a pancreatic tumor and/or lymph nodes (10). However, as to differentiating GP from carcinoma of the ampulla of Vater, many reports describe GP as often being visualized as a homogeneous, hypoechoic, solid tumor mass, though GP may reportedly be visualized as a heterogeneous, solid tumor. Since carcinoma of the ampulla of Vater is also visualized as a hypoechoic tumor mass, the differential diagnosis is difficult in patients showing no evidence of invasion. There is also a report noting that the CT findings of GP often show a plethoric solid tumor mass, but this is not spe- cific and making a diagnosis by CT is difficult. Since GP is a mixture of " 3 " cell types with different features, the CT findings vary according to the percentage of each cell type. A small GP is very difficult to distinguish from carcinoma of the ampulla of Vater. However, CT is considered to be the most useful modality for identifying lymph node metastasis in both GP and ampullary carcinoma cases. The ERCP findings of GP usually do not show dilation of either the pancreatic duct or the bile duct. Even in patients with a dilation of these ducts, jaundice is never observed and the dilatation is usually attributed to compression rather than invasion.

Carcinoma of the ampulla of Vater is also difficult to distinguish from GP in cases without invasion into the pancreatic duct and the bile duct in the early stage, as there are no findings suggestive of dilation in these cases.

Immunostaining is useful for the histopathological diagnosis. Okubo et al. reported the following: NSE was the most useful and synaptophysin the second most useful for epithelioid cells; S"100" was the most useful and NSE the second most useful for spindle cells; and synaptophysin was the most useful and NSE the second most useful for ganglionlike cells (11). The immunostaining results of our case were also uniformly positive (NSE-positive, S"100"-positive, and synaptophysin-positive). However, making a diagnosis based on the biopsy findings alone is difficult. The final diagnosis is generally made by an analysis after resection, since making a preoperative diagnosis is difficult.

There is no established therapy for GP, though a wide range of treatment methods from surgery to endoscopic resection are used (12). There is an increasing trend for pancreatoduodenectomy to be performed for patients with lymph node metastasis, whereas endoscopic resection is generally selected for those without lymph node metastasis, because GP is an inherently benign tumor. Furthermore, such 
patients are usually treated conservatively, because death from this tumor has not been reported in Japan. Regarding follow-up observation after treatment, there are some reports describing an association with lymph node metastasis, in which the epithelial component is considered to have a relatively high malignant potential because only the epithelial component has been observed in most metastatic lymph nodes $(13,14)$. In contrast, Sundararajan et al. reported that all "3" components were observed in lymph nodes with metastasis (15). Many reports describe no recurrence as having been observed in the period from " 6 " months after surgery until the end of an observation period as long as "91" months. However, more information on such cases should be accumulated in the future since there is also a report describing distant metastasis to the sternum " 3 " years after surgery and metastasis to mesenteric lymph nodes at " 11 " years (6). The indications for endoscopic therapy to treat ampullary tumors have been initially limited to adenoma only, but in recent years, there has been an expansion to tumors in situ and T"1" tumors. However, Yoon et al. reported that no recurrence was observed in patients with tumors in situ, whereas the recurrence rate was "18.2"\% (12/61) in those with $\mathrm{T}$ " 1 " tumors (16). Judging from these surgical observations, it might currently be appropriate that the indications for endoscopic therapy be limited to adenomas, tumors in situ, and T"1" tumors that have not reached the sphincter of Oddi. EUS and IDUS are used more widely than CT and magnetic resonance imaging (MRI) to assess local tumor progression. According to a report by Ito et al., the diagnostic performances of EUS vs. IDUS for T stage in " 40 " patients with ampullary tumors were " $62 " \%$ vs. " $86 " \%$ for adenoma and T"1" tumor, " 45 " $\%$ vs. " 64 " $\%$ for $\mathrm{T} " 2$ " tumors, and " 88 "\% vs. "75"\% for T"3"/“4", respectively, thus demonstrating a relatively low diagnostic performance for adenoma and T"1" lesions (17). Accordingly, endoscopic papillectomy, performed when detecting tumor progression is difficult, since it is not a treatment procedure but rather a total biopsy which is performed to make an accurate diagnosis and then select the most appropriate therapeutic strategy. In our case as well, the GP could be resected with the margin being negative, such that $\mathrm{s}$ cure by endoscopic resection was considered to have been achieved, though meticulous follow-up remains necessary.

\section{Conclusion}

We herein described a case with the coexistence of intraepithelial carcinoma in the superficial layer and GP in the deep portion, in which making an accurate diagnosis and selecting the most appropriate therapeutic strategy were challenging. Endoscopic resection resulted in a complete resection of both lesions and greatly contributed to maintaining our patient's quality of life.
The authors state that they have no Conflict of Interest (COI).

\section{References}

1. Scheithauer BW, Nora FE, LeChago J, et al. Duodenal gangliocytic paraganglioma. Clinicopathologic and immunocytochemical study of 11 cases. Am J Clin Pathol 86: 559-565, 1986.

2. Japanese Gastric Cancer Association. Japanese classification of gastric carcinoma: 3rd English edition. Gastric Cancer 14: 101112, 2011.

3. Dahl EV, Waugh JM, Dahlin DC. Gastrointestinal ganglioneuromas; brief review with report of a duodenal ganglioneuroma. Am J Pathol 33: 953-965, 1957.

4. Lin $\mathrm{D}, \mathrm{Hu} \mathrm{Y}$, Xing $\mathrm{X}$, et al. Pulmonary gangliocytic paraganglioma: a case report and review of the literature. Int J Clin Exp Pathol 7: 432-437, 2014.

5. Kepes JJ, Zacharias DL. Gangliocytic paragangliomas of the duodenum. A report of two cases with light and electron microscopic examination. Cancer 27: 61-67, 1971.

6. Witkiewicz A, Galler A, Yeo CJ, Gross SD. Gangliocytic paraganglioma: case report and review of the literature. J Gastrointest Surg 11: 1351-1354, 2007.

7. Okubo Y, Yokose T, Tuchiya M, et al. Duodenal gangliocytic paraganglioma showing lymph node metastasis: a rare case report. Diagn Pathol 5: 27, 2010.

8. Dookhan DB, Miettinen M, Finkel G, Gibas Z. Recurrent duodenal gangliocytic paraganglioma with lymph node metastases. Histopathology 22: 399-401, 1993.

9. Henry C, Ghalel-Mechaoui H, Bottero N, Pradier T, Moindrot H. [Gangliocytic paraganglioma of the pancreas with bone metastasis]. Ann Chir 128: 336-338, 2003 (in French, Abstract in English).

10. Smithline AE, Hawes RH, Kopecky KK, Cummings OW, Kumar S. Gangliocytic paraganglioma, a rare cause of upper gastrointestinal bleeding. Endoscopic ultrasound findings presented. Dig Dis Sci 38: 173-177, 1993.

11. Okubo Y, Wakayama M, Nemoto T, et al. Literature survey on epidemiology and pathology of gangliocytic paraganglioma. BMC Cancer 11: 1870, 2011.

12. Okubo $Y$, Nemoto T, Wakayama $M$, et al. Gangliocytic paraganglioma: a multi-institutional retrospective study in Japan. BMC Cancer 15: 269, 2015.

13. Saito J, Hirata N, Furuzono M, et al. A case of duodenal gangliocytic paraganglioma with lymph node metastasis. Nihon Shokakibyo Gakkai Zasshi 107: 639-648, 2010 (in Japanese, Abstract in English).

14. Uchida D, Ogawa T, Ueki $T$, et al. A case of gangliocytic paraganglioma with lymphoid metastasis. Nihon Shokakibyo Gakkai Zasshi 107: 1456-1465, 2010 (in Japanese, Abstract in English).

15. Sundararajan V, Robinson-Smith TM, Lowy AM. Duodenal gangliocytic paraganglioma with lymph node metastasis: a case report and review of the literature. Arch Pathol Lab Med 127: e139-e141, 2003.

16. Yoon YS, Kim SW, Park SJ, et al. Clinicopathologic analysis of early ampullary cancers with a focus on the feasibility of ampullectomy. Ann Surg 242: 92-100, 2005.

17. Ito K, Fujita N, Noda Y, et al. Preoperative evaluation of ampullary neoplasm with EUS and transpapillary intraductal US: a prospective and histopathologically controlled study. Gastrointest Endosc 66: 740-747, 2007.

The Internal Medicine is an Open Access article distributed under the Creative Commons Attribution-NonCommercial-NoDerivatives 4.0 International License. To view the details of this license, please visit (https://creativecommons.org/licenses/ by-nc-nd/4.0/).

(C) 2018 The Japanese Society of Internal Medicine

Intern Med 57: 2663-2668, 2018 